S5 Fig. The trend of early AMD risk by pack year separately for men and women.

Shown is the early AMD risk (on log scale) by pack year modelled by a sex-specific thin plate regression spline with the additional covariates sex, age (also modelled by a sex-specific thin plate regression spline) for A) men and B) women. The outcome is early AMD compared to controls (men: $n=131$ with early AMD, $n=1,116$ AMD-free; women: $n=141$ with early AMD, $\mathrm{n}=1,105$ AMD-free).

\title{
A: Men
}
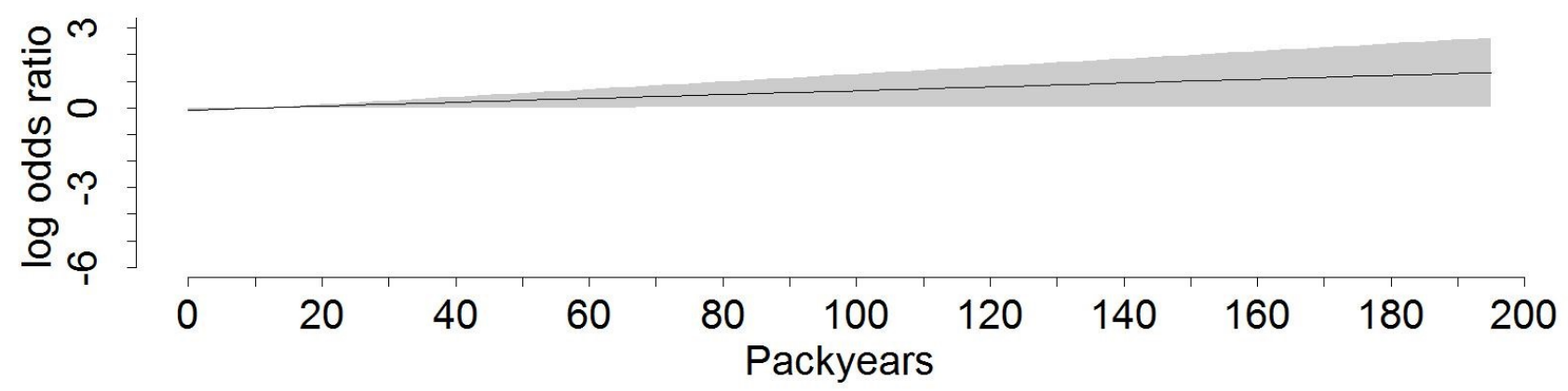

B: Women

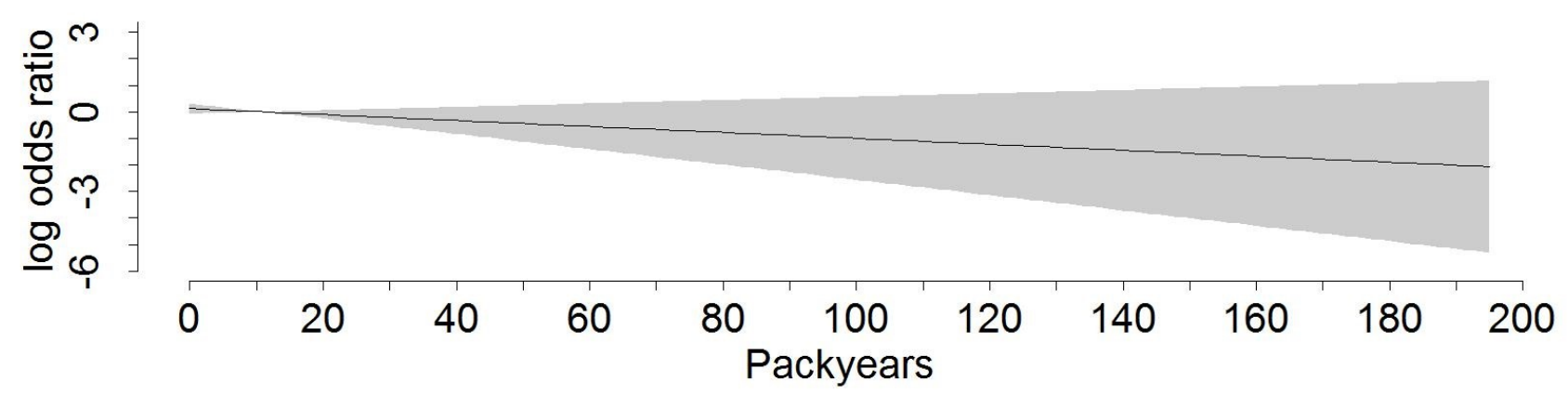

